\section{On the Relation Between State Feedback and Full Information Regulators in Nonlinear Singular $H_{\infty}$ Control}

\author{
A. Astolfi
}

\begin{abstract}
In this paper the relationship between state feedback control laws and full information control laws, i.e., control laws which make explicit use of the disturbance signals, is discussed in the framework of nonlinear singular $H_{\infty}$ control. In particular, it is shown that any (polynomial) full information control law can always be approximated by a state feedback control law, provided that a certain (arbitrarily small) amount of performance is sacrificed. Moreover, the obtained result is used to give alternative proofs and generalizations to some existing results in the area of global disturbance attenuation for nonlinear systems and to establish some new and interesting results regarding the problem of approximate model matching.
\end{abstract}

Index Terms -Almost disturbance attenuation, almost model matching, singular $H_{\infty}$ control.

\section{Introduction AND Problem Formulation}

Traditionally, state feedback $H_{\infty}$-control problems are solved under the assumption that the disturbances are also available for feedback; see [1] for the linear case and [2] for the nonlinear case. Moreover, it is possible to establish precise conditions, on the coefficient matrices which describe the system, under which the optimal $H_{\infty}$-control problem can be solved using pure state feedback control laws [2]. Such conditions are in general not fulfilled, hence the optimal control law depends also on the disturbances, i.e., the optimal feedback has a full information pattern. Full information control laws are extremely difficult to implement, as disturbances cannot be easily measured; thus the problem of approximating a full information control law by means of a state feedback law has a particular relevance, both from a theoretical and a practical point of view.

In this paper we deal with nonlinear systems described by equations of the form

$$
\begin{aligned}
& \dot{x}=f(x)+g(x) u+P\left(x, w, \cdots, w^{(k)}\right) \\
& z=h(x)
\end{aligned}
$$

where $k$ is a fixed (known) nonnegative integer, $w^{(i)}$ denotes the $i$ th-order time derivative of the disturbance vector $w$, and $P\left(x, w, w^{(1)}, \cdots, w^{(k)}\right) \in \mathcal{P}_{k}(x, w)$, where $\mathcal{P}_{k}(x, w)$ denotes the set of all vector valued functions with entries which are polynomial in the indeterminates $\left(w, w^{(1)}, \cdots, w^{(k)}\right)$ and such that $P(x, 0,0, \cdots, 0)=0$ for every $x$.

As usual, the first equation describes a plant defined in $\mathbb{R}^{n}$ with state $x$, control input $u \in \mathbb{R}^{m}$, and exogenous input $w \in \mathbb{R}^{r}$; whereas, the second equation defines a penalty variable $z \in \mathbb{R}^{s}$, which does not include the control input. We assume, without lack of generality, that $x=0$ is an equilibrium point for (1), i.e., $f(0)=0$, $P(0,0,0, \cdots, 0)=0$, and $h(0)=0$ and that the vector fields $f(x)$, $g(x)$, and $P\left(x, w, w^{(1)}, \cdots, w^{(k)}\right)$ are smooth vector fields, and $h(x)$ is a smooth mapping.

Manuscript received September 19, 1995; revised April 1, 1996.

The author is with the Department of Electrical and Electronic Engineering, Centre for Process Systems Engineering, Imperial College, London SW7 2BT U.K. (e-mail: a.astolfi@ic.ac.uk).

Publisher Item Identifier S 0018-9286(97)04276-1.
Throughout the paper we consider static-state feedback control laws modeled by equations of the form

$$
u=\alpha(x)
$$

or static, full information control laws

$$
u=\alpha(x)+\beta_{0}(x) w+\beta_{1}(x) w^{(1)}+\cdots+\beta_{n}(x) w^{(n)}
$$

or, finally, static, full information control laws

$$
u=M\left(x, w, w^{(1)}, \cdots, w^{(k)}\right)
$$

where $M\left(x, w, w^{(1)}, \cdots, w^{(k)}\right) \in \mathcal{P}_{k}(x, w)$. Moreover, we assume that the control laws are at least $k$ times differentiable (for some $k \geq 1$ ) with respect to $x$ and, to maintain the equilibrium, that $\alpha(0)=0$ and $M(0,0,0, \cdots, 0)=0$.

Remark 1: Notice that, for a physical reason, the order $k$ of the derivatives of the exogenous signal used in the control law should not exceed $n$.

The control laws (3) and (4) differ from standard full information control laws, as considered, e.g., in [2], as they also contain terms depending on the successive derivatives of the exogenous signal $w$. Hence, throughout the paper we restrict our attention to the class of exogenous signals $w$ which are, for almost every ${ }^{1} t \in[0, \infty), n$ times differentiable and such that there exist finite constants $k_{i}$ such that

$$
\int_{0}^{t}\left\|w^{(i)}(s)\right\| d s \leq k_{i} \int_{0}^{t}\|w(s)\| d s
$$

for every $i=1, \cdots, n$ and every $t>0$.

Remark 2: Note that every exogenous signal $w$ can be approximated to any arbitrary level of accuracy by an exogenous signal fulfilling the above assumptions.

For the class of nonlinear systems (1), we study the problem of global disturbance attenuation with internal stability. A rather standard way of handling such a problem is as follows (see [3]-[5] for more motivations).

Given a $\gamma>0$, find, if possible, a control law described by equations of the form (2), (3), or (4) and a smooth, real-valued, positive definite and proper function $V(x)$, such that along the trajectories of the closed-loop system, one has ${ }^{2}$

$$
H J I=\dot{V}-\gamma^{2}\|w\|^{2}+\|z\|^{2}<0
$$

and the closed-loop system (1) and (2), or (1)-(3), or (1)-(4), with $w(t) \equiv 0$, is globally asymptotically stable.

Remark 3: Note that the considered disturbance attenuation problem is referred to, in the literature, as a singular $H_{\infty}$-control problem; see, e.g., [5]-[7].

In the present paper we are not concerned with the problem of finding a solution for a certain global disturbance attenuation problem, as there already exists a complete characterization of such a problem. We assume that there exists a control law of the form (3) or (4) which solves, for a given $\gamma$, the considered disturbance attenuation problem, and we wonder whether there exists a control law of the form (2) which solves the problem for a certain $\tilde{\gamma}$. It is obvious that $\tilde{\gamma} \geq \gamma$.

The paper is organized as follows. In Section II we present the main results, i.e., a relation between the performance achievable by

${ }^{1}$ We say that a certain property is fulfilled for almost every $t \in[0, \infty)$ if the set where the property does not hold has a Lebesgue measure equal to zero.

${ }^{2}$ Throughout the paper we assume that the infinitesimal version of the dissipation inequalities are fulfilled for almost every $t \in[0, \infty)$. 
means of control laws described by equations of the form (3) or (4) and the performance achievable using control laws described by equations of the form (2); whereas, in Section III we give an auxiliary result which is instrumental in deriving some interesting applications of the main theorems. Finally, Section IV contains applications of the main results to some global control problems, i.e., the problems of almost disturbance decoupling, of singular $H_{\infty}$ control, and of almost model matching. Section V contains final remarks and an outline of future work.

\section{MAin Results}

The main result of this work can be summarized in the following statement.

Theorem 1: Consider (1). Assume that a full information control law described by equations of the form (3) solves, for a given $\gamma>0$, the problem of global disturbance attenuation with internal stability, i.e., there exists a smooth positive definite and proper function $V(x)$ such that, along the trajectories of the closed-loop system, one has

$$
\dot{V}-\gamma^{2}\|w\|^{2}+\|z\|^{2}<0
$$

and the closed-loop system, with $w(t) \equiv 0$, is globally asymptotically stable.

Then, for any $\gamma^{\star}>\gamma$ there exists a state feedback control law described by equations of the form (2) which solves the same problem.

Proof: To begin with, observe that from (7) we deduce

$$
\begin{aligned}
V_{x}(f(x)+g(x) \alpha(x) & \left.+P\left(x, w, \cdots, w^{(k)}\right)\right)+\|z\|^{2}-\gamma^{2}\|w\|^{2} \\
& +V_{x} g(x)\left(\beta_{0}(x) w+\cdots+\beta_{n}(x) w^{(n)}\right)<0 .
\end{aligned}
$$

Consider now a control law described by equations of the form

$$
u_{S F}=\alpha(x)+\beta_{0}(x) v_{0}+\beta_{1}(x) v_{1}+\cdots+\beta_{n}(x) v_{n}
$$

where the $v_{i}$ 's are functions of the state $x$ to be determined. Consider now the closed-loop system (1)-(9) and define, for a certain $\tilde{\gamma}>\gamma$ and for some $\epsilon_{i}>0$ (for all $i=1, \cdots, n$ ), the auxiliary function

$$
H J I_{S F}=\dot{V}-\tilde{\gamma}^{2}\|w\|^{2}-\epsilon_{1}^{2}\left\|w^{(1)}\right\|^{2}-\cdots-\epsilon_{n}^{2}\left\|w^{(n)}\right\|^{2}+\|z\|^{2} .
$$

Such a function is equal to

$$
\begin{aligned}
& V_{x}\left(f(x)+g(x) u_{S F}+P\left(x, w, w^{(1)}, \cdots, w^{(k)}\right)\right) \\
& -\tilde{\gamma}^{2}\|w\|^{2}-\epsilon_{1}^{2}\left\|w^{(1)}\right\|^{2}-\cdots-\epsilon_{n}^{2}\left\|w^{(n)}\right\|^{2}+\|z\|^{2} .
\end{aligned}
$$

Using (8), we obtain

$$
\begin{aligned}
& H J I_{S F}<V_{x} g(x)[ {\left[\beta_{0}(x)\left(v_{0}-w\right)+\cdots+\beta_{n}(x)\left(v_{n}-w^{(n)}\right)\right] } \\
&-\left(\tilde{\gamma}^{2}-\gamma^{2}\right)\|w\|^{2}-\epsilon_{1}^{2}\left\|w^{(1)}\right\|^{2}-\cdots \epsilon_{n}^{2}\left\|w^{(n)}\right\|^{2} .
\end{aligned}
$$

Then, setting

$$
v_{0}=v_{0}^{\star}=-\frac{1}{2\left(\tilde{\gamma}^{2}-\gamma^{2}\right)} \beta_{0}^{T}(x) g^{T}(x) V_{x}^{T}
$$

and

$$
v_{i}=v_{i}^{\star}=-\frac{1}{2 \epsilon_{i}^{2}} \beta_{i}^{T}(x) g^{T}(x) V_{x}^{T}
$$

for all $i=1, \cdots, n$, we obtain, after completing the squares

$$
H J I_{S F}<-\left(\tilde{\gamma}^{2}-\gamma^{2}\right)\left\|w-v_{0}^{\star}\right\|^{2}-\sum_{i=1}^{n} \epsilon_{i}^{2}\left\|w^{(i)}-v_{i}^{\star}\right\|^{2} \leq 0 .
$$

Hence, using (5), we have, for almost every $t \geq 0$

$$
\begin{aligned}
V_{x}\left(f(x)+g(x) u_{S F}^{\star}\right. & \left.+P\left(x, w, w^{(1)}, \cdots, w^{(k)}\right)\right) \\
& +\|z\|^{2}-\left(\gamma^{\star}\right)^{2}\|w\|^{2}<0
\end{aligned}
$$

where

$$
u_{S F}^{\star}=\alpha(x)+\beta_{0}(x) v_{0}^{\star}+\beta_{1}(x) v_{1}^{\star}+\cdots+\beta_{n}(x) v_{n}^{\star}
$$

and

$$
\gamma^{\star}=\sqrt{\tilde{\gamma}^{2}+k_{1}^{2} \epsilon_{1}^{2}+\cdots+k_{n}^{2} \epsilon_{n}^{2}} .
$$

Finally, from the arbitrariness of $\tilde{\gamma}>\gamma$ and $\epsilon_{i}>0$, the conclusion directly follows.

Using the same arguments, it is possible to prove the following generalization of Theorem 1 .

Theorem 2: Consider (1). Assume that a full information control law described by equations of the form (4), with $M\left(x, w, w^{(1)}, \cdots, w^{(n)}\right) \in \mathcal{P}_{n}(x, w)$, solves, for a given $\gamma>0$, the problem of global disturbance attenuation with internal stability.

Then, for any $\gamma^{\star}>\gamma$ there exists a state feedback control law described by equations of the form (2) which solves the same problem.

Remark 4: By (10) and (11), it is obvious that the approximating control law has a high-gain nature.

\section{An AuXiliary Result}

In this Section we present a simple Lemma which will be useful to develop some applications of Theorems 1 and 2. It is a modified version of a result in [8].

Lemma 1: Consider a single-input/single-output system described by equations of the form

$$
\begin{aligned}
\dot{z} & =f_{0}\left(z, \eta_{1}\right)+p_{0}\left(z, \eta_{1}\right) w \\
\dot{\eta}_{1} & =\eta_{2} \\
\dot{\eta}_{2} & =\eta_{3} \\
& \vdots \\
\dot{\eta}_{r-1} & =\eta_{r} \\
\dot{\eta}_{r} & =u+M_{r}\left(z, \eta_{1}, \cdots, \eta_{r}, w, \cdots, w^{(r-1)}\right) \\
y & =\eta_{1}
\end{aligned}
$$

where $w \in \mathbb{R}, M_{r}\left(z, \eta_{1}, \cdots, \eta_{r}, w, w^{(1)}, \cdots, w^{(r-1)}\right) \in \mathcal{P}_{r-1}(z$, $\left.\eta_{1}, \cdots, \eta_{r}, w\right)$ and $f_{0}(0,0)=0$. Suppose:

H1) there exist a smooth real-valued function

$$
\eta_{1}=v(z)
$$

with $v(0)=0$ and a smooth real-valued function $V(z)$, which is positive definite and proper, such that for some $\gamma>0$

$$
V_{z}\left(f_{0}(z, v(z))+p_{0}(z, v(z)) w\right)-\gamma^{2}\|w\|^{2}<0
$$

for every nonzero $z$.

Then:

1) for the same $\gamma$, there exist a positive definite and proper function $W\left(z, \eta_{1}, \cdots, \eta_{r}\right)$ and a full information control law described by equations of the form

$$
\begin{aligned}
u= & \alpha\left(z, \eta_{1}, \cdots, \eta_{r}\right) \\
& -M_{r}\left(z, \eta_{1}, \cdots, \eta_{r}, w, w^{(1)}, \cdots, w^{(r-1)}\right)
\end{aligned}
$$

such that, along the trajectories of the closed-loop system, (6) is fulfilled;

2) for every $\tilde{\gamma}>\gamma$ there exist a positive definite and proper function $W\left(z, \eta_{1}, \cdots, \eta_{r}\right)$ and a state feedback control law such that, along the trajectories of the closed-loop system, (6) with $\tilde{\gamma}$ instead of $\gamma$, is fulfilled. 
Proof: The proof of 1) follows a standard backstepping procedure (see, e.g., [8]), hence it is omitted; whereas, 2) is a direct consequence of 1) and of Theorem 2.

\section{FURTHER RESULTS}

\section{A. Almost Disturbance Decoupling for Nonlinear Systems}

From Theorems 1 and 2 it is possible to deduce the following interesting result which relates the problem of disturbance decoupling with stability to the problem of almost disturbance decoupling with stability (see [9] for a precise definition of such control problems).

Corollary 1: Consider a system of the form (1). Assume that there exists a full information control law of the form (3) or (4) which solves, globally, the problem of disturbance decoupling with stability.

Then, there exists a state feedback control law, described by equations of the form (2), which solves, globally, the problem of almost disturbance decoupling with stability.

A class of nonlinear systems for which the problem of global almost disturbance decoupling with stability is solvable is described by equations of the form

$$
\begin{aligned}
\dot{z} & =f_{0}\left(z, \xi_{1}\right)+p_{0}\left(z, \xi_{1}\right) w \\
\dot{\xi}_{1} & =\xi_{2}+P_{1}\left(z, \xi_{1}, w\right) \\
\dot{\xi}_{2} & =\xi_{3}+P_{2}\left(z, \xi_{1}, \xi_{2}, w\right) \\
& \vdots \\
\dot{\xi}_{r-1} & =\xi_{r}+P_{r-1}\left(z, \xi_{1}, \xi_{2}, \cdots, \xi_{r-1}, w\right) \\
\dot{\xi}_{r} & =u+P_{r}\left(z, \xi_{1}, \xi_{2}, \cdots, \xi_{r-1}, \xi_{r}, w\right) \\
y & =\xi_{1}
\end{aligned}
$$

where $u \in \mathbb{R}$ is the control variable, $w \in \mathbb{R}$ the disturbance input, $y \in \mathbb{R}$ the regulated output, and, for all $i=1, \cdots, r$

$$
P_{i}\left(z, \xi_{1}, \cdots, \xi_{i}, w\right) \in \mathcal{P}_{0}\left(z, \xi_{1}, \cdots, \xi_{i}, w\right) .
$$

As a matter of fact, the following result, which is a generalization of a result in [9] and [5], holds.

Proposition 1: Consider a nonlinear single-input/single-output system described by equations of the form (15) with $f_{0}(0,0)=0$ and $p_{0}(z, 0)=0$. Assume that the system $\dot{z}=f_{0}(z, 0)$ is globally asymptotically stable.

Then the problem of almost disturbance decoupling with stability is globally solvable by means of a state feedback control law.

Proof: Consider the subsystem

$$
\begin{aligned}
\dot{z} & =f_{0}\left(z, \xi_{1}\right)+p_{0}\left(z, \xi_{1}\right) w \\
\dot{\xi}_{1} & =u_{1}+P_{1}\left(z, \xi_{1}, w\right)
\end{aligned}
$$

and observe that it is a special case of (12) with $r=1$. Moreover, as $\left.p_{0}(\zeta, 0)=0, \mathrm{H} 1\right)$ of Lemma 1 reduces to

$$
V_{\zeta} f_{0}(\zeta, 0)-\gamma^{2}\|w\|^{2}<0 .
$$

Hence, from the global asymptotic stability of the system

$$
\dot{\zeta}=f_{0}(\zeta, 0)
$$

and in view of the converse Lyapunov theorem, we conclude that there exists a smooth real-valued function $V(\zeta)$ which is positive definite and proper, such that

$$
V_{\zeta} f_{0}(\zeta, 0)<0
$$

for every nonzero $\zeta$.

Moreover, by Lemma 1, for all $\gamma_{1}>0$ there exist a positive definite and proper function $W_{1}\left(z, \xi_{1}\right)$ and a state feedback control law $u_{1}=u_{1}\left(z, \xi_{1}\right)$ which solve the problem of global disturbance attenuation with internal stability for the subsystem (16).

Repeating the same arguments $n-1$ times, we conclude the claim.

\section{B. Singular $H_{\infty}$ Control for Triangular Systems}

Consider again a nonlinear system of the form (15). If $p_{0}(z, 0) \neq 0$ the following result, which is a slight generalization of a result in [8], holds.

Proposition 2: Consider a nonlinear single-input/single-output system described by equations of the form $(15)$ with $f_{0}(0,0)=0$. Assume that there exist a smooth real-valued function $\xi_{1}=v(z)$ and a smooth real-valued function $V(x)$, which is positive definite and proper, such that for a certain $\gamma>0$

$$
V_{z}\left(f_{0}(z, v(z))+p_{0}(z, v(z)) w\right)-\gamma^{2}\|w\|^{2}<0
$$

for every nonzero $z$.

Then for every $\tilde{\gamma}>\gamma$, the problem of global disturbance attenuation with stability is solvable by means of a state feedback control law.

Proof: The proof is similar to that of Proposition 1 and is, therefore, omitted.

\section{Approximate Model Matching with Stability}

Again, from Theorems 1 and 2, it is possible to deduce the following interesting result which relates the problem of asymptotic model matching with stability to the problem of almost model matching with stability (see [10] and [11] for a precise definition of such control problems).

Corollary 2: Consider a system of the form (1). Assume that there exists a full information control law of the form (3) or (4) which solves, globally, the problem of asymptotic model matching with stability.

Then, there exists a state feedback control law, described by equations of the form (2), which solves, globally, the problem of almost model matching with stability.

As in Section IV-A, we now consider a special class of systems for which the problem of asymptotic model matching with stability is solvable using full information control laws described by equations of the form (3) or (4).

Consider a nonlinear system, with input $u \in \mathbb{R}$ and output $y \in \mathbb{R}$, modeled by equations of the form

$$
\begin{aligned}
\dot{z} & =f_{0}\left(z, \xi_{1}\right) \\
\dot{\xi}_{1} & =\xi_{2} \\
\dot{\xi}_{2} & =\xi_{3} \\
& \vdots \\
\dot{\xi}_{r-1} & =\xi_{r} \\
\dot{\xi}_{r} & =u \\
y & =\xi_{1}
\end{aligned}
$$

with $f_{0}(0,0)=0$, and a linear asymptotically stable reference model described by equations of the form

$$
\begin{aligned}
\dot{\zeta} & =A \zeta+b w \\
y_{R} & =c \zeta
\end{aligned}
$$

where $\zeta \in \mathbb{R}^{n_{M}}$ is the state of the model, $w \in \mathbb{R}$ is the input to the model, and $y_{R} \in \mathbb{R}$ is the reference output. Let $s$ be the relative degree of the model (18), i.e.,

$$
c b=c A b=\cdots=c A^{s-1} b=0 .
$$


Define an error $e$ as the difference between the real output $y$ and the reference output $y_{R}$

$$
e=y-y_{R} \text {. }
$$

Following the approach in [11] we say that the problem of approximate model matching with stability is globally solvable if there exist a smooth state feedback or full information control law and a smooth real-valued function $V$, which is positive definite and proper, such that along the trajectories of the closed-loop system one has for a certain $\gamma>0$

$$
\dot{V}-\gamma^{2}\|w\|^{2}+\|e\|^{2}<0
$$

and the closed-loop system with $w \equiv 0$ is globally asymptotically stable. If $\gamma$ can be chosen arbitrarily small, we are dealing with a problem of almost model matching with stability.

We are now ready to prove the following result.

Proposition 3: Consider a nonlinear system described by equations of the form (17) and a linear, asymptotically stable reference model described by equations of the form (18). Let $s$ be the relative degree of the model and assume $s=n_{M}$. Assume, moreover, that there exist a smooth real-valued function $\xi_{1}=v(z)$ and a smooth real-valued function $V(z)$, which is positive definite and proper, such that

$$
V_{z} f_{0}(z, v(z))<0
$$

for every nonzero $z$, i.e., the system $\dot{z}=f_{0}(z, v(z))$ is globally asymptotically stable.

Then, there exists a dynamic, full information control law described by equations of the form

$$
\begin{aligned}
\dot{\zeta} & =A \zeta+b w \\
u & =\alpha\left(\zeta, z, \xi_{1}, \xi_{2}, \cdots, \xi_{r}\right)
\end{aligned}
$$

which solves, globally, the problem of almost model matching with stability.

Proof: We break the proof into two steps. In the former, through a global coordinates transformation we show that the composite system (17), (18) can be described by equations of the form (15) with a state vector which includes the error $e$ and with regulated output the error $e$. In the latter we prove that, for the transformed system, H1) of Lemma 1 can be fulfilled for any $\gamma>0$, implying that (19) holds for any $\gamma>0$.

1) Consider the globally defined coordinates transformation

$$
\begin{aligned}
\zeta^{\prime} & =\zeta \\
z^{\prime} & =z \\
e_{1} & =\xi_{1}-y_{R} \\
e_{2} & =\xi_{2}-\dot{y}_{R} \\
& \vdots \\
e_{n_{M}} & =\xi_{n_{M}}-y_{R}^{\left(n_{M}-1\right)} \\
e_{n_{M}+1} & =\xi_{n_{M}+1} \\
& \vdots \\
e_{r} & =\xi_{r} .
\end{aligned}
$$

Simple calculations show that in the new coordinates, the system with input $w$ and output $e=e_{1}$ is described by equations of the form

$$
\begin{aligned}
\dot{\zeta}^{\prime} & =A \zeta^{\prime}+b w \\
\dot{z}^{\prime} & =f_{0}\left(z^{\prime}, e_{1}+c \zeta^{\prime}\right) \\
\dot{e}_{1} & =e_{2} \\
\dot{e}_{2} & =e_{3} \\
& \vdots \\
\dot{e}_{n_{M}} & =e_{n_{M}+1}-w \\
\dot{e}_{n_{M}+1} & =e_{n_{M}+2} \\
& \vdots \\
\dot{e}_{r} & =u \\
e & =e_{1}
\end{aligned}
$$

i.e., by equations of the form (15) with $z=\operatorname{col}\left(\zeta^{\prime}, z^{\prime}\right)$ and $\eta_{i}=e_{i}$.

2) Consider now the zero dynamics of (20), i.e.,

$$
\begin{aligned}
& \dot{\zeta}^{\prime}=A \zeta^{\prime}+b w \\
& \dot{z}^{\prime}=f_{0}\left(z^{\prime}, e_{1}+c \zeta^{\prime}\right) .
\end{aligned}
$$

The evaluation of (14) appearing in H1) of Lemma 1 with

$$
W\left(\zeta^{\prime}, z^{\prime}\right)=\zeta^{\prime T} X \zeta^{\prime}+V\left(z^{\prime}\right)
$$

for some $X>0^{3}$ and $e_{1}=-c \zeta^{\prime}+v\left(z^{\prime}\right)$ yields

$$
\begin{aligned}
H\left(\zeta^{\prime}, z^{\prime}, w\right)= & 2 w^{T} b^{T} X \zeta^{\prime}+\zeta^{\prime T}\left(A^{T} X+X A\right) \zeta^{\prime} \\
& +V_{z^{\prime}} f_{0}\left(z^{\prime}, v\left(z^{\prime}\right)\right)-\gamma^{2}\|w\|^{2} .
\end{aligned}
$$

Hence, after simple calculations we obtain

$$
\begin{aligned}
H\left(\zeta^{\prime}, z^{\prime}, w\right) \leq & \zeta^{\prime T}\left(A^{T} X+X A+X \frac{b b^{T}}{\gamma^{2}} X\right) \zeta^{\prime} \\
& +V_{z^{\prime}} f_{0}\left(z^{\prime}, v\left(z^{\prime}\right)\right) .
\end{aligned}
$$

To prove the claim we have to show that for every $\gamma>0$, there exists a positive definite matrix $X$ such that

$$
A^{T} X+X A+X \frac{b b^{T}}{\gamma^{2}} X<0 .
$$

Consider the ARE

$$
A^{T} X+X A+X \frac{B B^{T}}{\gamma^{2}} X=0
$$

with $B \in \mathbb{R}^{n_{M} \times n_{M}}$ nonsingular and such that

$$
B B^{T}>b b^{T} \text {. }
$$

It is readily seen that a positive definite solution $X$ of the ARE (23) satisfies the ARI (22). Consider now the Lyapunov equation

$$
Y A^{T}+A Y+\frac{B B^{T}}{\gamma^{2}}=0
$$

which, by stability of $A$, has a symmetric positive definite solution $Y$ for every $\gamma>0$. It is readily seen that $X=Y^{-1}>$ 0 solves (23) and fulfills (22). Hence, the proof is complete.

\footnotetext{
${ }^{3}$ It must be noted that the smooth function $W\left(\zeta^{\prime}, z^{\prime}\right)$ is positive definite and proper.
} 


\section{CONCLUSION}

We have studied the relationship between state feedback control laws and full information control laws. In particular, we have shown that any control law which can be expressed as a polynomial in the exogenous signal and its successive derivatives can be approximated, to any degree of accuracy, by means of a state feedback control law. Some interesting implications of this result have also been presented. Among them, a relation between the problem of disturbance decoupling and the problem of almost disturbance decoupling has been provided. Moreover, solutions to the problems of almost disturbance decoupling, of singular $H_{\infty}$ control, and of almost model matching with stability have been derived. It must be noticed that the approach used in this paper not only yields alternative proofs for some of the results in [9], [5], and [8] but also allows us to generalize such results. The author believes that the proposed approach can be useful in dealing with approximate problems. Finally, further studies to characterize the problem of approximate regulation with stability are in progress and will be reported elsewhere.

\section{REFERENCES}

[1] A. A. Stoorvogel, The $H_{\infty}$ Control Problem: A State Space Approach. Englewood Cliffs, NJ: Prentice-Hall, 1992.

[2] A. Isidori, "Feedback control of nonlinear systems," in 1st European Contr. Conf., 1991.

[3] A. Isidori and A. Astolfi, "Disturbance attenuation and $H_{\infty}$-control via measurement feedback in nonlinear systems," IEEE Trans. Automat. Contr., vol. 37, pp. 1283-1293, Sept. 1992.

[4] A. J. Van der Schaft, " $L_{2}$-gain analysis of nonlinear systems and nonlinear state feedback $H_{\infty}$ control," IEEE Trans. Automat. Contr., vol. 37, pp. 770-784, June 1992.

[5] R. Marino, W. Respondek, A. J. Van der Schaft, and P. Tomei, "Nonlinear $H_{\infty}$ almost disturbance decoupling," Syst. Contr. Lett., vol. 23, pp. $159-168$, Sept. 1994.

[6] W. C. A. Maas and A. J. Van der Schaft, "Singular nonlinear $H_{\infty}$ optimal control by state feedback," in 33rd Conf. Decision Contr., Orlando, FL, 1994.

[7] A. Astolfi, "Singular $H_{\infty}$ control for nonlinear systems," Int. J. Robust Nonlinear Contr., to appear.

[8] A. Isidori, "A note on almost disturbance decoupling for nonlinear minimum phase systems," in Symp. Nonlinear Contr. Syst. Design, Tahoe City, NV, 1995.

[9] R. Marino, W. Respondek, and A. J. Van der Schaft, "Almost disturbance decoupling for single-input single-output nonlinear systems," IEEE Trans. Automat. Contr., vol. 34, pp. 1013-1017, Sept. 1989.

[10] A. Isidori, Nonlinear Control Systems, 2nd ed. New York: Springer Verlag, 1989.

[11] L. Guzzella and A. Isidori, "Approximate matching of nonlinear systems via nonlinear $H_{\infty}$ methods," in Symp. Nonlinear Contr. Syst. Design, Bordeaux, France, 1992.

\section{Synthesis of Positive Real $\mathcal{H}_{2}$ Controllers}

\author{
J. C. Geromel and P. B. Gapski
}

\begin{abstract}
This paper describes a synthesis procedure for strictly positive real observer-based controllers. In particular, we address the standard $\mathcal{H}_{2}$-optimal control problem with the additional constraint on the strict positive realness of the controller. A suboptimal version of this problem is shown to be a convex optimization problem, and a linear matrix inequality-based solution is provided. One example illustrates the main results.
\end{abstract}

Index Terms-Continuous-time systems, linear matrix inequality, positive real controllers, robust control.

\section{INTRODUCTION}

In recent years the control community has drawn a lot of attention to problems related to the robustness of control systems against exogenous perturbations and model uncertainties. Currently, there are several interesting results which, apart from theoretical value, help the control designer to best adapt his designs to user needs.

Most of the work done related to robustness of linear systems relies on important theorems from nonlinear analysis, such as the small-gain theorem and the passivity theorem. It is well known that these theorems are valuable tools in both analysis and design of robust control systems. This comes from the fact that stability is guaranteed once one of these theorems is satisfied [1]. Moreover, it is possible to show that small-gain and passivity results are in some sense dual [2].

Consider the passivity theorem. It states that if we connect in negative feedback two square passive operators, one of them being strictly passive, then the closed-loop system is stable. This theorem is readily recognized as a powerful tool in robustness analysis. Let $\mathcal{P}$ be the set of all positive real plants. If a strictly positive real controller is designed for the nominal plant $P_{0} \in \mathcal{P}$, then closedloop system stability is preserved whenever $P_{0}$ is replaced by any other plant $P \in \mathcal{P}$ [1]. In other words, the controller so designed (taking into account only the nominal plant information) stabilizes all plants $P \in \mathcal{P}$.

In some applications (see the control of a flexible structure provided in [3]), the nominal system to be controlled has the key property that $P_{0} \in \mathcal{P}$, while the system uncertainties represented by the finiteorder truncation are such that the actual plant is $P \in \mathcal{P}$. Therefore, in such cases, if a strictly positive real controller is used to close the loop, then the uncertain feedback system will be stable. Furthermore, for any kind of perturbations such that the uncertain open-loop plant remains positive real, any strictly positive real controller guarantees robust stability. Notice that if the controller, based on the nominal plant, is not strictly positive real, then it may exist $P \in \mathcal{P}$ such that the closed-loop system is unstable. These arguments show the importance of strictly positive real control design.

Recent works [4] have studied the synthesis of internally stable controllers imposing positivity to the closed-loop system. However, no special care has been given to select among all feasible controllers

Manuscript received April 11, 1995; revised November 15, 1995 and August 3, 1996. This work was supported by grants from "Fundação de Amparo à Pesquisa do Estado de São Paulo-FAPESP" and "Conselho Nacional de Desenvolvimento Científico e Tecnológico-CNPq"-Brazil.

The authors are with LAC-DT, School of Electrical Engineering, UNICAMP, 13081-970, Campinas, SP, Brazil (e-mail: geromel@dt.fee.unicamp.br).

Publisher Item Identifier S 0018-9286(97)04289-X. 